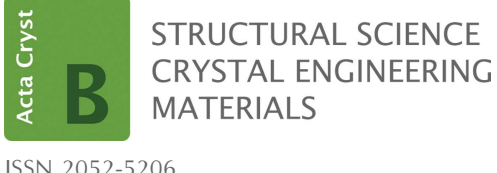

ISSN 2052-5206

\section{Natural Quasicrystals: The Solar System's Hidden Secrets. By Luca Bindi. Springer Briefs in Crystallography, Vol. 1, Springer, 2020, x+89 pp. Softcover. Price EUR 51.99. ISBN 978-3-030-45676-4}

\author{
Ron Lifshitz* \\ Raymond and Beverley Sackler School of Physics and Astronomy, Tel Aviv University, Tel Aviv, 69978, Israel. \\ *Correspondence e-mail: ronlif@tau.ac.il
}

Crack open a geode, or pick up a shiny rock near a natural water stream, and there is a good chance you will see crystals. But the chances that these will be quasicrystals are close to nil. Why is it that quasicrystalline minerals appear to be so rare? Is it surprising, or should we be surprised that they exit at all? Natural Quasicrystals: The Solar System's Hidden Secrets, by Luca Bindi, tells the story of the search for quasicrystals that occur naturally as minerals, and proposes a plausible explanation for how, and surprisingly where, they may have formed.

Allow me to begin with a quick recap of post-Shechtman terminology. A crystal is a structure possessing long-range order in the positions of its atoms, indicated by the presence of Bragg peaks in its diffraction diagram (p. 928 in IUCr, 1992; Lifshitz, 2007). Crystals may or may not be periodic. Quasicrystals are quasiperiodic crystals (Levine \& Steinhardt, 1986) that are explicitly aperiodic (Lifshitz, 2003). They lack periodicity, but they are crystals nevertheless. Icosahedral or decagonal crystals cannot be periodic. Cubic crystals, on the other hand, may or may not be periodic. The simplest icosahedral crystals, those whose Bragg peaks are indexed by six integers, are classified into three Bravais classes (Rokhsar et al., 1987): simple (or primitive) icosahedral (si), bodycentered icosahedral (bci), and face-centered icosahedral (fci).

How surprising is the existence of quasicrystals in general? When Dan Shechtman discovered the first quasicrystal (Shechtman et al., 1984) it shattered the prevailing paradigm that all crystals were periodic, and ushered a bona fide Kuhnian scientific revolution (Kuhn, 1962). Today, almost four decades later, aperiodic crystals are found almost everywhere, and while their realization in new contexts and novel physical systems is exciting as ever, and often provides important insight, it is hardly surprising. It is in this context that one should consider the search for quasicrystalline minerals.

Years ago, a group of scientists led by Paul Steinhardt, who would eventually become the author's collaborators, embarked on a quest to find such minerals, by searching through scientific databases and issuing a public call to curators of mineral collections worldwide. The search eventually led to a $4 \mathrm{~mm}$ rock in the collection of Bindi's home institute, the Museo di Storia Naturale of the Università degli Studi di Firenze (in Italy). The sample originated from the Khatyrka region of the Kamchatka peninsula in Russia. It consisted of light-colored material on its exterior, surrounding a dark substance, consisting mainly of khatyrkite and cupalite (metallic alloys of copper and aluminium), with tiny granules of the sought-after quasicrystal, which was given the mineral name 'icosahedrite' for its icosahedral point-group symmetry.

Ensuing analysis established that the granules were face-centered icosahedral (fci) crystals with the familiar composition of $\mathrm{Al}_{63} \mathrm{Cu}_{24} \mathrm{Fe}_{13}$ - the first thermodynamically stable quasicrystal ever discovered (Tsai et al., 1987, Calvayrac et al., 1990). Further analysis of the sample showed traces of stishovite, a polymorph of $\mathrm{SiO}_{2}$ that usually forms at very high pressures and temperatures, as well as a distribution of oxygen isotopes that indicated that the sample might be of extraterrestrial origin. These and other details led to the hypothesis that the quasicrystalline mineral was formed through a high-pressure and high-temperature shock, generated by a hypervelocity impact of asteroids in outer space - a truly spectacular event that may have taken place as early as when the solar system itself was forming, around 4.5 billion years ago, hence the title of the book. 
In the summer of 2011, to establish their hypothesis, Bindi and his collaborators went on an expedition to recover additional fragments of the Khatyrka meteorite. A number of new minerals were identified within the recovered fragments, among them three interesting aluminium-rich alloys with about half the iron content of icosahedrite or less. These were a decagonal quasicrystal with composition $\mathrm{Al}_{71} \mathrm{Ni}_{24} \mathrm{Fe}_{5}$, which was given the mineral name 'decagonite'; a periodic approximant of a decagonal quasicrystal, with composition $\mathrm{Al}_{34} \mathrm{Ni}_{9} \mathrm{Fe}_{2}$, which was named 'proxidecagonite'; and another fci quasicrystal with composition $\mathrm{Al}_{61} \mathrm{Cu}_{32} \mathrm{Fe}_{7}$, which was left unnamed and labeled i-phase II.

The decagonal AlNiFe crystal, also one of the first stable quasicrystals to be discovered (Tsai et al., 1989), is known to lose its thermodynamic stability below around $847^{\circ} \mathrm{C}$ (Grushko et al., 1996), consistent with the scenario of hightemperature shock-production, followed by rapid cooling. An icosahedral quasicrystal with a precise composition of $\mathrm{Al}_{61} \mathrm{Cu}_{32} \mathrm{Fe}_{7}$ had not been reported before, but one with a nearby composition of $\mathrm{Al}_{62} \mathrm{Cu}_{29} \mathrm{Fe}_{9}$ was observed at $660^{\circ} \mathrm{C}$ (Zhang et al., 2005), as noted by the author, and reconfirmed recently at $700^{\circ} \mathrm{C}$ (Zhu et al., 2020). The author chose to identify i-phase II as being different from the known icosahedral AlCuFe phase, hailing it as the first quasicrystal found in nature, prior to being discovered in the laboratory. This may well be true, as I may have missed something, but without additional structural analysis I would think that such a claim is premature.

All of the above is covered in chapters 3 and 4 of the book. Chapter 5 reproduces experimental work by the author and his collaborators, reconfirming the stability of quasicrystals under the increase of static pressure (Krauss \& Steurer, 2004). Chapter 6 depicts experimental studies in collaboration with Paul Asimow, who simulated the shock that may have been produced by a collision of asteroids. Thin slices of the relevant minerals from the meteorite, including aluminium-copper alloys, were sandwiched together and fired upon with a highspeed projectile. In some cases, the impact indeed produced icosahedral and decagonal microcrystals, supporting the hypothesis that shocks may have been responsible for the formation of icosahedrite and decagonite.

I am not certain that Natural Quasicrystals achieves its intended goal as the inaugural volume of the new series, 'Springer Briefs in Crystallography.' The core of the book, as summarized above, reproduces previously published work by the author and his collaborators. In addition, chapters 1 and 2 offer minimal background and motivation, while chapters 7 and 9, each only two pages long, provide little in the way of an adequate discussion or summary for the book. I expected the book, as brief as it is, to benefit from having been written in hindsight, and to paint a broad picture, while providing some synthesis, along with a critical assessment of the research and its implications.

There is no argument that the author's findings are important, but the book follows a somewhat exaggerated narrative that overstates their significance. Unlike the author, I recall that by the mid-1990s it was well established that quasicrystals were stable forms of matter, no less stable than periodic crystals. There were, and still are, debates regarding the origin of their stability. But already then, no one seriously studying quasicrystals claimed they were 'inherently delicate, metastable oddities that must be synthesized under highly controlled laboratory conditions.' By the late 1990s it was demonstrated, again and again, that growing quasicrystalline alloys was no more difficult than growing periodic alloys (Canfield \& Fisher, 2001). By no means was there any need for a confirmation of the mere stability of quasicrystals to come from the unearthing of a quasicrystalline mineral. Steinhardt's presentation of icosahedrite, at the 2010 International Conference on Quasicrystals (Steinhardt \& Bindi, 2011), was well received, but it did not take anyone by surprise (Dubois \& Lifshitz, 2011). Most were generally pleased and curious. Some doubted the speculative arguments. But, no one thought it would be impossible for quasicrystalline minerals to exist improbable or rare, perhaps, but not impossible.

Almost as odd is the author's question whether Nature had 'beaten us to the punch' by forming quasicrystals long before they were 'synthesized' in a lab. Surely, the degree of human intervention, when growing crystals in the lab, is quite minor. The real magic occurs after ingredients are in place, external conditions are set, and a quasicrystal - rather than a periodic crystal - forms spontaneously from the melt through a phase transition. Nature performs this magic, whether it happens in the lab or in outer space. Shechtman took liquid aluminiummanganese and rapidly cooled it. Nature did the rest. His Nobel Prize citation reads: 'for the discovery of quasicrystals', and not for an 'experimental breakthrough' that yielded 'a piece of synthetic material ... with the symmetry of an icosahedron.' As a skilled electron microscopist, he was able to rule out twinning, and to infer that what Nature had produced for him was a new (metastable) icosahedral phase with longrange order, which we now call a quasicrystal. In fact, (stable) icosahedral $\mathrm{AlCuFe}$ was synthesized long ago (Bradley \& Goldschmidt, 1939), but recognized by Tsai as being a quasicrystal only after Shechtman's discovery (Tsai, 2003).

So, why is it that quasicrystalline minerals appear to be so rare? The recipe calls for collecting all the right ingredients, removing any possible contaminants, and creating the proper thermodynamic conditions. If you think about it, it is only fitting that the first minerals identified as being quasicrystalline are also among the first thermodynamically stable quasicrystals to be discovered in the lab - those consisting of very common metals: aluminium, copper, nickel, and iron. But, as noted by the author, from a geological standpoint, the presence of metallic aluminium in minerals - periodic and aperiodic alike - is in itself quite puzzling and rare. This is because aluminium tends to oxidize, and requires highly reducing conditions to even form metallic alloys. The author mentions only three or four additional sites in the world where aluminium-bearing alloys were found, none of which has been identified as a quasicrystal, as of yet.

Thus, the simplest quasicrystals contain metallic aluminium, which is rare in minerals, and seem to require extreme extraterrestrial conditions to form. Other families of quasi- 
crystals, with less common metals such as the $\mathrm{ZnMgRE}$ family (where RE stands for rare earth elements such as Y, Gd, Tb, Dy, Ho, Er), or the Cd-based family (Tsai, 2003), are not even considered as mineral candidates in the book. Nevertheless, Bindi speculates that quasicrystals may be much more common in the universe than we expect, and that it is very likely that other quasicrystalline minerals will be found soon, 'now that the first discovery is settled and well accepted by the scientific community.' I do hope he is right, and I look forward to new findings, but I do not believe that the apparent rareness of quasicrystalline minerals is the sole result of scientific prejudice.

\section{Acknowledgements}

The author is grateful to Alan Goldman for his helpful remarks.

\section{Funding information}

The following funding is acknowledged: Israel Science Foundation (grant No. 1667/16).

\section{References}

Bradley, A. J. \& Goldschmidt, H. J. (1939). J. Inst. Met. 65, 403418.
Calvayrac, Y., Quivy, A., Bessière, M., Lefebvre, S., CornierQuiquandon, M. \& Gratias, D. (1990). J. Phys. Fr. 51, 417-431.

Canfield, P. C. \& Fisher, I. R. (2001). J. Cryst. Growth, 225, 155161.

Dubois, J.-M. \& R. Lifshitz, R. (2011). Philos. Mag. 91, 2971.

Grushko, B., Lemmerz, U., Fischer, K. \& Freiburg, C. (1996). Phys. Status Solidi. A, 155, 17.

IUCr (1992). Acta Cryst. A48 922-946.

Krauss, G. \& Steurer, W. (2004). In High-Pressure Crystallography, edited by A. Katrusiak \& P. McMillan, pp. 521-526. Dordrecht: Springer.

Kuhn, T. S. (1962). The Structure of Scientific Revolutions. University of Chicago Press.

Levine, D. \& Steinhardt, P. J. (1986). Phys. Rev. B, 34, 596-616.

Lifshitz, R. (2003). Found. Phys. 33, 1703-1711.

Lifshitz, R. (2007). Z. Kristallog. 222, 313.

Rokhsar, D. S. N. D., Mermin, N. D. \& Wright, D. C. (1987). Phys. Rev. $B, 35,5487-5495$.

Shechtman, D. I., Blech, D., Gratias, D. \& Cahn, J. W. (1984). Phys. Rev. Lett. 53, 1951-1953.

Steinhardt, P. J. \& Bindi, L. (2011). Philos. Mag. 91, 2421-2426.

Tsai, A.-P. (2003). Acc. Chem. Res. 36, 31-38.

Tsai, A.-P. A., Inoue, A. \& Masumoto, T. (1987). Jpn. J. Appl. Phys. 26, L1505-L1507.

Tsai, A.-P. A., Inoue, A. \& Masumoto, T. (1989). Mater. Trans. JIM, 30, 150-154.

Zhang, L., Schneider, J. \& Lück, R. (2005). Intermetallics, 13, 11951206.

Zhu, L., Soto-Medina, S., Cuadrado-Castillo, W., Hennig, R. G. \& Manuel, M. V. (2020). Mater. Des. 185, 108186. 